\title{
Elaboração, avaliação mercadológica e análise sensorial de pudim gelado sem lactose
}

\author{
Elaboration, market evaluation and sensory analysis of lactose-free iced pudding \\ Elaboración, evaluación de mercado y análisis sensorial de pudín helado sin lactosa
}

Recebido: 30/04/2021 | Revisado: 05/05/2021 | Aceito: 20/05/2021 | Publicado: 08/06/2021

Fernanda de Oliveira Vieira

ORCID: https://orcid.org/0000-0001-6050-5500

Universidade Comunitária da Região de Chapecó, Brasil

E-mail: fernanda.vieira@unochapeco.edu.br

Suelem Vieira dos Santos

ORCID: https://orcid.org/0000-0002-0432-3179

Universidade Comunitária da Região de Chapecó, Brasil

E-mail: suelem.santos@unochapeco.edu.br

Caroline Tombini

ORCID: https://orcid.org/0000-0002-7543-1003

Universidade Comunitária da Região de Chapecó, Brasil

E-mail: caroline.tombini@unochapeco.edu.br

Priscila Favero Moratelli

ORCID: https://orcid.org/0000-0002-9203-7956

Universidade Comunitária da Região de Chapecó, Brasil E-mail: prifavero@unochapeco.edu.br

Francisco Roberto da Silva Machado Junior

ORCID: https://orcid.org/0000-0002-0960-2927

Universidade Federal do Rio Grande, Brasil

E-mail: franciscojr_ea@yahoo.com.br

Micheli Zanetti

ORCID: https://orcid.org/0000-0001-5218-7522

Universidade Comunitária da Região de Chapecó, Brasil

E-mail: eng.miche@unochapeco.edu.br

Francieli Dalcanton

ORCID: https://orcid.org/0000-0003-0065-1279

Universidade Comunitária da Região de Chapecó, Brasil

E-mail: fdalcanton@unochapeco.edu.br

\begin{abstract}
Resumo
O desenvolvimento de novos produtos se faz necessário para suprir a demanda do consumidor, que cada vez mais apresenta anseios e necessidades específicas. Nesse sentido, o mercado de produtos zero lactose se apresenta em constante crescimento, visando atender essa demanda. Estima-se que a cada 10 brasileiros, 7 são intolerantes, sendo que para alguns, a intolerância está presente desde os primeiros momentos de vida. Diante disso e buscando avaliar o atual cenário mercadológico no âmbito de produtos sem lactose, aplicou-se um questionário online e foi realizada a elaboração de uma sobremesa do tipo pudim gelado sem lactose, o qual passou por avaliação sensorial e de intenção de compra como método de estudo para investigar o potencial mercadológico deste produto. O questionário online resultou em 521 respostas e apontou que $22,8 \%$ eram intolerantes a lactose, enquanto $71,2 \%$ não possuíam nenhuma intolerância. A análise sensorial do pudim gelado sem lactose apresentou uma aceitação acima de $70 \%$ em todos os atributos na escala hedônica e $92 \%$ dos voluntários responderam que certamente ou provavelmente comprariam o produto. Conclui-se assim, que o pudim zero lactose desenvolvido agradou os avaliadores tanto intolerantes, quanto não intolerantes, apresentando uma alternativa promissora no cenário de produtos sem lactose pois, além disso, apresentou custo similar a um pudim tradicional.
\end{abstract}

Palavras-chave: Intolerância; Sobremesa sem lactose; Análise sensorial.

\begin{abstract}
The development of new products is necessary to meet consumer demand, which increasingly presents specific needs and desires. In this sense, the market for zero lactose products is constantly growing, in order to meet this demand. It is estimated that for every 10 Brazilians, 7 are intolerant, and for some, intolerance has been present since the first moments of life. In view of this and seeking to assess the current market scenario in the context of lactose-free products, an online questionnaire was applied and a dessert of the frozen pudding type without lactose was carried out, which underwent sensory evaluation and purchase intention as a method. study to investigate the market potential of this product. The online questionnaire resulted in 521 responses and pointed out that $22.8 \%$ were lactose intolerant, while $71.2 \%$ had no intolerance. The sensory analysis of iced pudding without lactose showed an acceptance above $70 \%$ in all attributes on the hedonic scale and $92 \%$ of the volunteers answered that they would certainly or probably
\end{abstract}


buy the product. It is concluded, therefore, that the developed zero lactose pudding pleased both intolerant and nonintolerant evaluators, presenting a promising alternative in the scenario of lactose-free products because, in addition, it has a similar cost to a traditional pudding.

Keywords: Intolerance; Lactose-free dessert; Sensory analysis.

\section{Resumen}

El desarrollo de nuevos productos es necesario para satisfacer la demanda del consumidor, que presenta cada vez más necesidades y deseos específicos. En este sentido, el mercado de productos sin lactosa está en constante crecimiento, con el fin de satisfacer esta demanda. Se estima que de cada 10 brasileños, 7 son intolerantes, y para algunos, la intolerancia ha estado presente desde los primeros momentos de la vida. Ante esto y buscando valorar el escenario actual del mercado en el ámbito de los productos sin lactosa, se aplicó un cuestionario online y se realizó un postre del tipo de pudín sin lactosa, el cual se sometió a evaluación sensorial e intención de compra como método. .Estudio para investigar el potencial de mercado de este producto. El cuestionario en línea resultó en 521 respuestas y señaló que el $22,8 \%$ eran intolerantes a la lactosa, mientras que el $71,2 \%$ no tenía intolerancia. El análisis sensorial del pudín helado sin lactosa mostró una aceptación superior al 70\% en todos los atributos en la escala hedónica y el $92 \%$ de los voluntarios respondieron que seguro o probablemente comprarían el producto. Se concluye, por tanto, que el pudín cero lactosa elaborado agradó a los evaluadores, tanto intolerantes como no intolerantes, presentando una alternativa prometedora en el escenario de los productos sin lactosa porque, además, presenta un costo similar a un pudín tradicional.

Palabras clave: Intolerancia; Postre sin lactosa; Análisis sensorial.

\section{Introdução}

O desenvolvimento de novos produtos é uma atividade de vital importância para a sobrevivência da maioria das empresas e a renovação contínua de produtos é uma política generalizada no âmbito empresarial. Essas inovações também devem abranger os públicos com restrições alimentares, como é o caso dos consumidores com intolerância à lactose. De acordo com Matias (2015), o desenvolvimento de produtos é um processo de negócio cada vez mais crítico devido a internacionalização dos mercados, o aumento da diversidade de produtos e a redução do seu ciclo de vida, sendo assim, novos produtos buscam atender segmentos específicos de mercado, incorporando novas tecnologias e se adequando a novos padrões e restrições legais.

$\mathrm{O}$ mercado brasileiro de produtos alimentícios com baixo teor de lactose, alimentos destinados à população intolerante à lactose e mesmo o público que busca por alimentos isentos de lactose por preferência, carece de diversificação (Silva et al., 2020a). Cabe ressaltar que os produtos lácteos sem lactose são capazes de fornecer os nutrientes essenciais presentes nos produtos lácteos regulares, como o cálcio e as vitaminas, para aqueles que não são capazes de digerir a lactose (Dekker; Koenders; \& Bruins, 2019). Portanto, a indústria de alimentos, visando atender às expectativas de diferentes públicos e nichos de mercado, tem investido fortemente em inovação (Silva et al., 2020b), visto que, o processo de desenvolvimento de produtos necessita de investimento, pesquisa, planejamento e ferramentas de apoio para que as empresas atinjam as suas metas através do lançamento de novos produtos no mercado consumidor.

Nos últimos anos, é notável o crescimento do número de produtos lácteos com teor reduzido ou sem a presença de lactose. Neste contexto, a introdução destes produtos tem o objetivo de promover o consumo de lácteos também por pessoas com intolerância à lactose, evitando consequências indesejáveis posteriormente (Gille et al., 2018). A lactose tem grande importância na indústria de alimentos, pois através da sua fermentação por microrganismos específicos, obtém-se o ácido lático, sendo esse processo utilizado para a produção de diversos derivados como iogurte, leite fermentado, bebida láctea, queijo, entre outros. Formada pela união dos monossacarídeos glicose e galactose, a lactose é o principal açúcar presente no leite (Lima; do Nascimento; \& de Farias, 2019). Por isso, ela se torna componente fundamental em muitas formulações de alimentos.

Diversos estudos tem sido apresentados para o desenvolvimento de produtos sem lactose, visando atender as necessidades deste público consumidor, avaliando a elaboração de diversos alimentos, tais como queijos (Da Silva et al., 
2020), bolo tipo muffin (Alves; Moro; Clareto; Clerici; \& Moraes, 2020) e brownie (Dutra; Pante; Reck; Bánkuti; \& Madrona, 2020).

Ainda, a literatura aponta que a intolerância à lactose é muito comum na população e que grande parte dela pode ser considerada intolerante a esse dissacarídeo (Branco; Dias; Fernandes; Berro; \& Simioni, 2018). Este tipo de intolerância é a sensibilidade metabólica alimentar mais comum, afetando $60-70 \%$ das pessoas em todo o mundo. Níveis inadequados da enzima lactase resultam em desconforto abdominal, inchaço, gases e diarreia porque a lactose não digerida no cólon é fermentada por bactérias (Luthy; Larimer; \& Freeborn, 2017).

Além dos desconfortos físicos causados pela doença, ela também prejudica o convívio social, já que há restrição alimentar considerada, tornando-se difícil o hábito alimentar da família, pois diversos alimentos devem ser omitidos da dieta. Para as crianças isto é ainda mais complicado, pois esses têm dificuldade de entender a proibição de certas guloseimas (Silva; Costa; \& Giugliani, 2016).

Segundo Uggioni e Fagundes (2006), o tratamento da intolerância à lactose consiste basicamente na retirada ou diminuição desse açúcar da dieta, o que leva ao desaparecimento progressivo dos sintomas. Uma das grandes preocupações com a redução da lactose da alimentação é a garantia do fornecimento de quantidade apropriada de proteínas, cálcio, riboflavina e vitamina $\mathrm{D}$, cuja maior fonte é o leite e seus derivados.

Neste sentido, visando suprir a necessidade deste mercado que demanda por produtos específicos, a indústria alimentícia tem investido esforços para levar ao consumidor produtos que sejam, além de nutritivos, de degustação prazerosa. Assim, o objetivo deste estudo foi desenvolver um pudim gelado sem lactose e avaliar a sua aceitação sensorial, além de realizar uma avaliação mercadológica do perfil do consumidor.

\section{Metodologia}

O estudo se classifica como survey, descritivo e experimental (Gil, 2010).

\subsection{Avaliação mercadológica do perfil do consumidor}

Para determinação de um panorama do número de pessoas intolerantes à lactose foi elaborado um questionário via plataforma online (Google Forms) que pode ser observado no Quadro 1, sendo que este foi avaliado e aprovado pelo Comitê de Ética em Pesquisa Envolvendo Seres Humanos da Unochapecó, com CAAE (Certificado de Apresentação de Apreciação Ética) sob o número de protocolo 34758820.8.0000.0116. O questionário foi enviado via e-mail para professores e alunos da Universidade Comunitária da Região de Chapecó - Unochapecó e via grupos de Whatsapp, recebendo respostas durante 14 dias.

As perguntas foram elaboradas com o objetivo de alcançar o maior número de pessoas, buscando compreender o cenário de oferta de produtos zero lactose no mercado, a frequência com que este tipo de alimento é consumido e as dificuldades que o consumidor encontra com relação à oferta destes produtos. 
Quadro 1 - Questionário online de avaliação mercadológica.

\section{1) Qual a sua idade?}

( ) 18 a 20 anos; ( ) 21 a 25 anos; ( ) 26 a 35 anos; ( ) 36 a 45 anos; ( ) 46 a 50 anos; ( ) mais de 50 anos
2) Qual sua profissão?
3) Onde você reside?

4) Qual seu nível de escolaridade?

( ) $1^{\circ}$ Grau incompleto; ( ) $1^{\circ}$ Grau completo; ( ) $2^{\circ}$ Grau incompleto; ( ) $2^{\circ}$ Grau completo; ( ) Superior incompleto; ( ) Superior completo
5) Gênero?
( ) Masculino ( ) Feminino
( ) Outro:

6) Você tem alguma intolerância alimentar?

( ) Lactose ( ) Glúten ( ) Proteína do leite ( ) Não tenho intolerância ( ) Outras:

7) Você consome produtos zero lactose?

( ) $\operatorname{Sim}$ ( ) Não

8) Se você é intolerante a lactose você utiliza enzimas digestivas antes de ingerir alimento que contenha lactose?

( ) Sim ( ) Não

9) Com que frequência você consome alimento tipo sobremesa zero lactose?

( ) todos os dias () uma vez por semana ( ) de 2 a 3 vezes por semana

( ) 1 a 3 vezes por mês () nunca

10) Você encontra com facilidade produtos com essas características (sobremesa, com custo relativamente baixo, zero lactose) no mercado? ( ) Sim （ ) Não

11) Você considera que produtos zero lactose apresentam custo elevado?

( ) $\operatorname{Sim}$ ( ) Não

12) Em relação ao custo de produtos zero lactose, você optaria por um alimento zero lactose, mas que tenha um valor agregado maior?

( ) Sim ( ) Não

13) Você gostaria de ter uma opção de sobremesa zero lactose?

( ) Sim ( ) Não ( ) Indiferente

14) Alguém do seu convívio tem influência ou necessita fazer consumo desse tipo de alimento (zero lactose)?

( ) Sim ( )Não

Fonte: Autores.

\subsection{Elaboração do pudim gelado sem lactose}

O pudim gelado foi elaborado a partir de ingredientes que já dispõem de composição zero lactose, adquiridos em comércio local e estes são apresentados na Tabela 1. A elaboração dos produtos, bem como a análise sensorial destes foi realizada nos laboratórios de Tecnologia de Alimentos e Análise Sensorial da Universidade Comunitária da Região de Chapecó - Unochapecó. Foram realizadas 3 receitas iguais, obtendo-se, com essas quantidades, 54 unidades de pudim zero lactose, sendo que cada pudim pesou em média, $30 \mathrm{~g}$. 
Tabela 1 - Ingredientes e quantidades utilizadas na produção de uma receita de pudim gelado zero lactose

\begin{tabular}{ccc}
\hline Ingredientes & Quantidade & Marca \\
\hline Leite condensado zero lactose & $790 \mathrm{~g}$ & Piracanjuba \\
Creme de leite zero lactose & $400 \mathrm{~g}$ & Piracanjuba \\
Açúcar Refinado & $250 \mathrm{~g}$ & União \\
Suco de Limão Siciliano & $150 \mathrm{~mL}$ & - \\
Gelatina sem sabor & $12 \mathrm{~g}$ & Dr. Oetker \\
\hline
\end{tabular}

Fonte: Autores.

Para a elaboração do pudim gelado, foram realizadas as etapas conforme a descrição no fluxograma apresentado na Figura 1.

Figura 1 - Fluxograma de processamento do pudim gelado sem lactose.

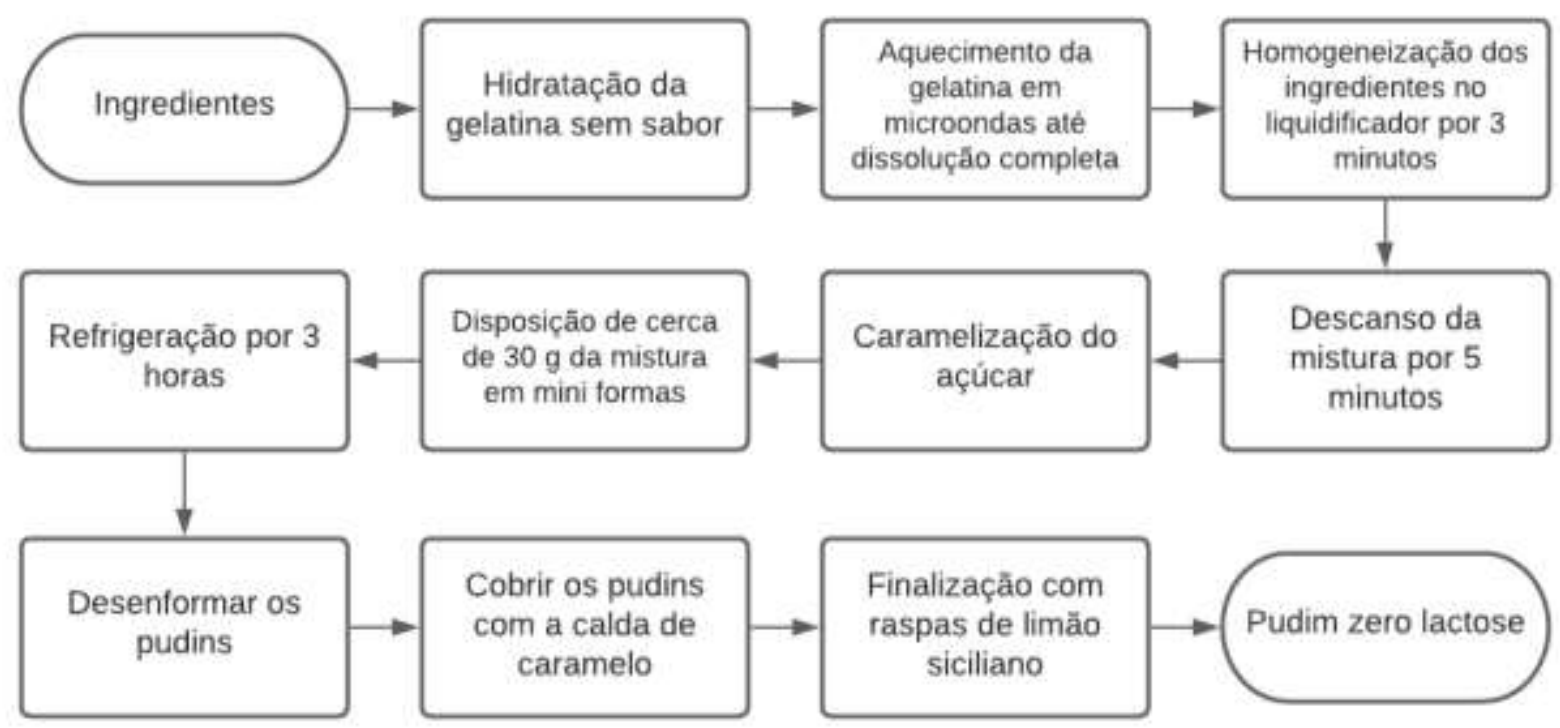

Fonte: Autores.

Conforme observado na Figura 1, primeiramente hidratou-se a gelatina em pó em $75 \mathrm{~mL}$ de água e a mistura foi levada ao micro-ondas (marca Electrolux) por 15 segundos para completa homogeneização. Em seguida, foram adicionados ao liquidificador (marca Mundial) o creme de leite, o leite condensado, a gelatina dissolvida já em temperatura ambiente, e por fim, o suco de limão sem sementes. Os ingredientes foram misturados por 3 minutos em velocidade baixa até obter-se uma massa homogênea. Posteriormente, a massa permaneceu em repouso por cerca de 5 minutos em temperatura ambiente enquanto preparou-se a calda de açúcar.

Separadamente, preparou-se a calda com a adição de $250 \mathrm{~g}$ de açúcar refinado e $50 \mathrm{~mL}$ de água em uma panela. Esta foi levada ao fogo médio e permaneceu sob agitação contínua até que a calda apresentou coloração caramelo.

Em seguida, despejou-se a mistura do pudim preparada anteriormente em forma de silicone com formato de mini pudim, com capacidade para $30 \mathrm{~mL}$ em cada cavidade, e esta foi levada ao refrigerador (marca Electrolux) por 3 horas em temperatura de aproximadamente $6{ }^{\circ} \mathrm{C}$.

Depois, os pudins foram desenformados e acomodados em pratos individuais (Figura 2), verteu-se a calda de açúcar por cima de cada pudim e adicionou-se raspas de limão siciliano. 
Figura 2 - Imagem de pudim gelado sem lactose após desenformar.

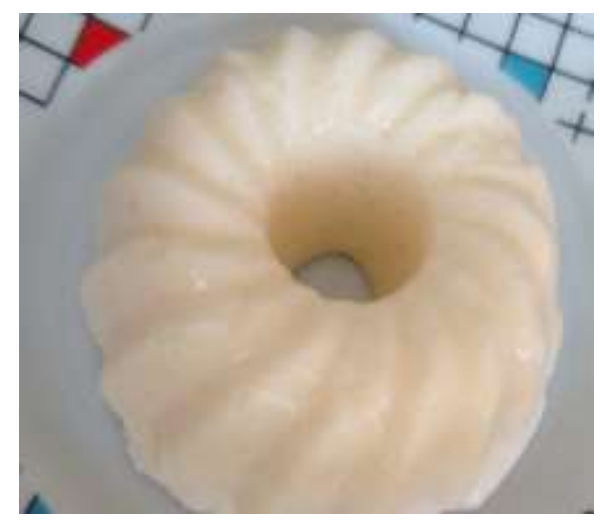

Fonte: Autores.

Realizou-se também um comparativo no que diz respeito aos custos de produção de um pudim tradicional (com lactose) frente a um pudim sem lactose. Desta forma, elaborou-se uma tabela com os valores por ingrediente de forma unitária e considerando a quantidade necessária de cada um para a produção de uma receita do produto. Os valores dos ingredientes foram consultados em um site de um supermercado local, de acordo com as marcas dos produtos utilizados na produção dos pudins.

\subsection{Análises Sensoriais}

Para este estudo, utilizou-se o teste de aceitação sensorial por escala hedônica, em que o indivíduo expressa o grau de gostar ou de desgostar de um determinado produto, de forma globalizada ou em relação a um atributo específico. As escalas mais utilizadas são as de 7 e 9 pontos, que contêm os termos definidos situados entre "gostei muitíssimo" e "desgostei muitíssimo", contendo pontos intermediários com os termos “nem gostei; nem desgostei” (Instituto Adolfo Lutz, 2008). O projeto foi avaliado e aprovado pelo Comitê de Ética em Pesquisa Envolvendo Seres Humanos da Unochapecó, com CAAE (Certificado de Apresentação de Apreciação Ética) sob o número de protocolo 34758820.8.0000.0116.

O estudo contou com 50 avaliadores, sendo eles acadêmicos, professores e colaboradores da Unochapecó. Foi analisada também a intenção de compra, perfil do avaliador, e se é ou não intolerante à lactose. Serviu-se ao avaliador uma amostra de pudim sem lactose de aproximadamente $30 \mathrm{~g}$. Junto a cada amostra servida, o avaliador recebeu um copo de água em temperatura ambiente para limpar o paladar. Na Figura 3 pode ser observado a amostra servida.

Figura 3 - Imagem que representa a amostra de pudim sem lactose servida aos avaliadores na análise sensorial.



Fonte: Autores. 
Para a avaliação de aceitação por escala hedônica, o avaliador demonstrou o quanto ele gostou ou desgostou do pudim gelado sem lactose quanto aos atributos de odor, aparência, sabor, textura e impressão global, através do teste afetivo de aceitabilidade baseado em escala hedônica estruturada de nove pontos, conforme Quadro 2. Os avaliadores também indicaram a intenção de compra do produto por meio de escala de cinco pontos (certamente compraria, provavelmente compraria, tenho dúvidas se compraria, provavelmente não compraria e certamente não compraria), assinalando a sua opção na mesma ficha de avaliação.

Quadro 2 - Ficha de avaliação sensorial e intenção de compra do pudim zero lactose.

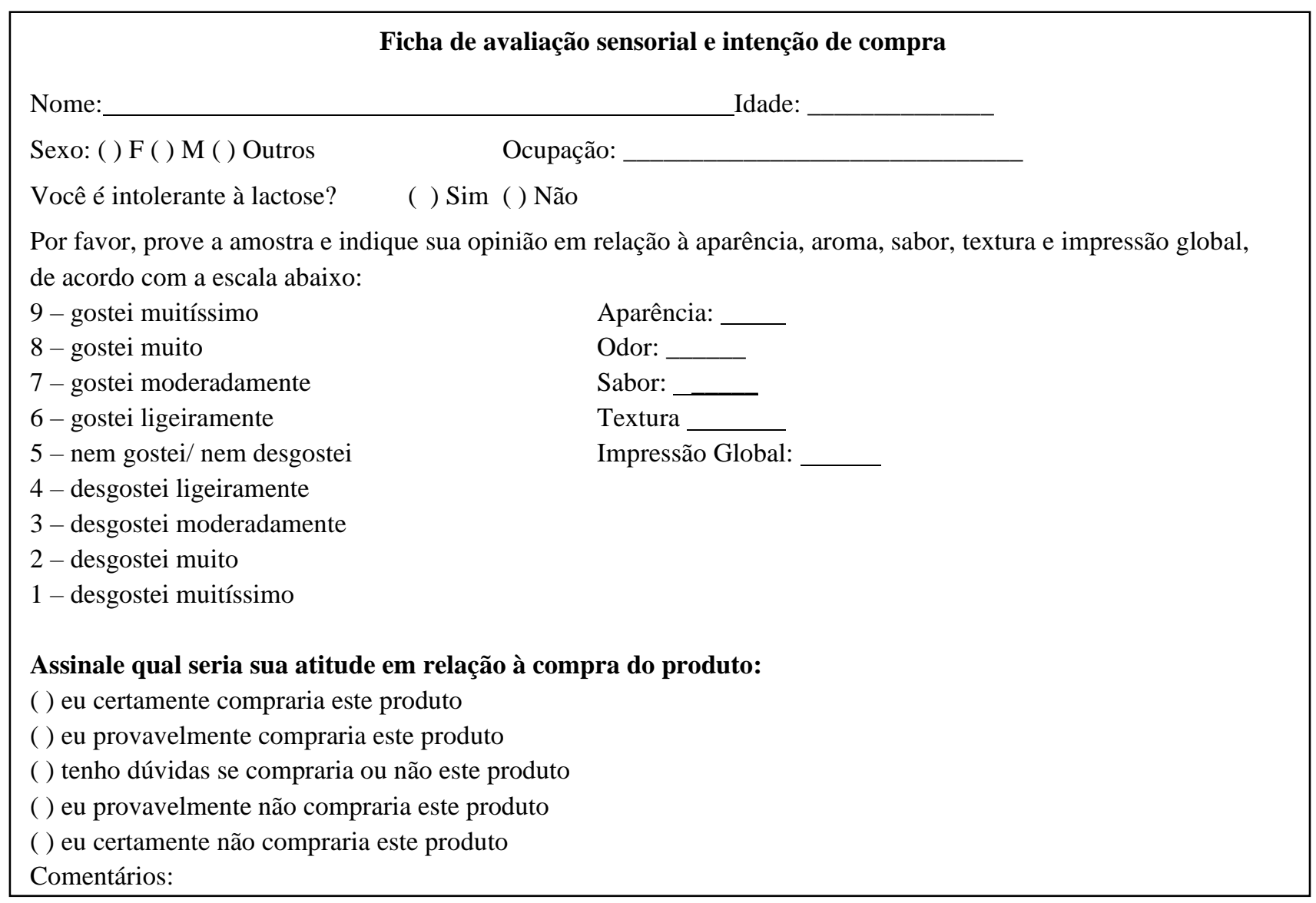

Fonte: Adaptado de Instituto Adolfo Lutz (2008).

\section{Resultados e Discussão}

O questionário online para avaliação de aceitação mercadológica obteve 521 respostas. As respostas apontaram que $78,3 \%$ dos respondentes foram mulheres, com idade entre 18 e 20 anos, e $90 \%$ das pessoas que responderam à pesquisa tinham ensino superior completo ou incompleto.

Quanto ao percentual de intolerantes, $22,8 \%$ eram intolerantes a lactose, enquanto $71,2 \%$ declararam não possuir nenhuma intolerância, conforme observa-se no Figura 4. 
Figura 4 - Percentual de pessoas que declaram não possuir nenhum tipo de intolerância ou de ser intolerantes à lactose, glúten, proteína do leite ou outras.

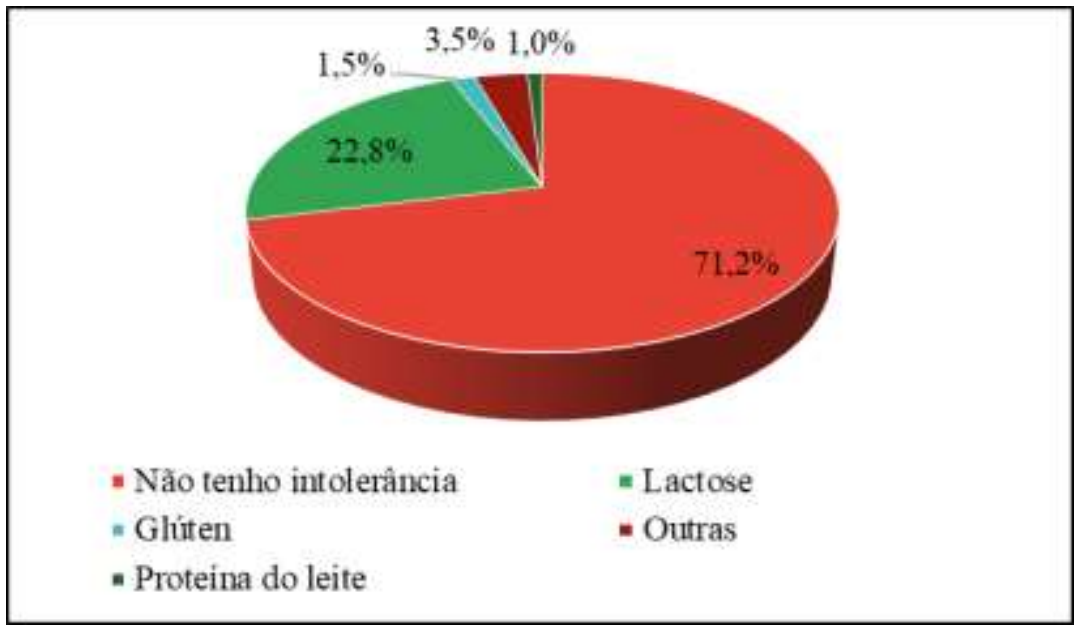

Fonte: Autores.

Eiki e colaboradores (2015) obtiveram resultado semelhante em seu estudo, quando avaliaram o percentual de intolerantes, em que $14 \%$ dos respondentes da pesquisa declararam possuir intolerância à lactose. Este número apresenta-se inferior à média apontada para a população mundial, que é de cerca de 60-70\% (Luthy; Larimer; \& Freeborn, 2017). Isso pode ser explicado pelo fato de que muitas pessoas não possuem conhecimento da sua condição intolerante, pois Eiki e colaboradores evidenciaram que $98 \%$ do público participante da pesquisa declarou não possuir alergias alimentares, porém, tal resposta pode apresentar certo grau de complexidade visto que requer o conhecimento acerca do tema. De forma similar, observou-se neste estudo, cujo objetivo principal com a pesquisa foi de evidenciar dados a respeito da intolerância à lactose, que conceitos relacionados a alergias e sensibilidade, como a sensibilidade ao glúten, carecem de esclarecimentos com embasamento na legislação.

Quando perguntado se consomem alimentos zero lactose, 56,8\% disseram não consumir e 27,3\% responderam consumir esses alimentos com frequênca de 1 a 3 vezes por semana, como mostra o Figura 5.

Figura 5 - Frequência de consumo de alimentos do tipo zero lactose.

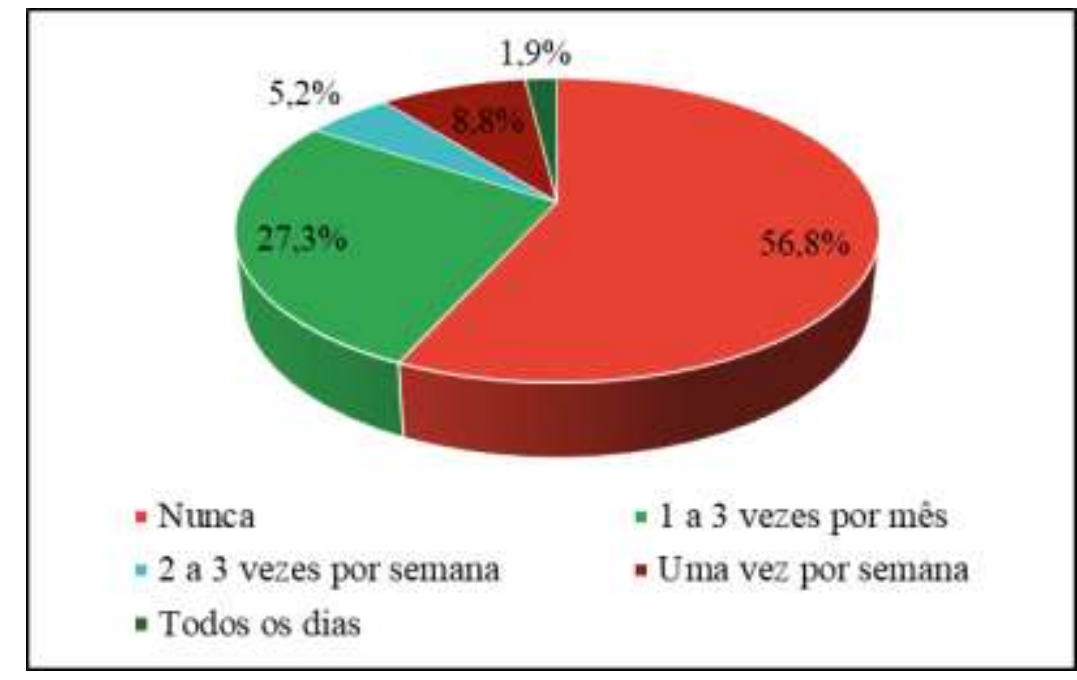

Fonte: Autores. 
Quanto ao grau de dificuldade para encontrar este tipo de alimento, 53,2\% disseram não encontrar com facilidade produtos do tipo zero lactose nos mercados e $85,2 \%$ consideram que alimentos zero lactose têm um custo mais elevado se comparado com produtos convencionais. Ainda assim, 48,6\% responderam que comprariam produtos zero lactose.

Ao questionar sobre a variedade desse tipo de alimento, 53,4\% responderam que gostariam de ter mais opções de alimento tipo zero lactose e 45,3\% são indiferentes. Dantas, Verruck e Prudencio (2019) destacam alternativas para o público que apresenta má digestão da lactose, em que o mercado disponibiliza produtos como lácteos fermentados, os queijos, o doce de leite, o leite condensado e os leites com reduzido teor de lactose.

Buscou-se avaliar ainda no presente estudo, sobre a condição de influência de outras pessoas no consumo de produtos zero lactose ou se convivem com pessoas intolerantes, e observou-se que 58,3\% dos respondentes disseram ter em seu convívio pessoas com intolerância e/ou que lhes influenciam na ingestão de comidas zero lactose.

Este formato de pesquisa já esteve em foco em estudos anteriores, quando comprovadamente a comodidade de poder responder o questionário no domicilio virtualmente, não ter o trabalho e o gasto de tempo para preparar e enviar o questionário postal aumenta a taxa de respostas dos questionários virtuais (Faleiros et al., 2016). Dessa forma, foi possível se ter uma percepção e analisar os índices de intolerantes, e também a acessibilidade destes participantes aos produtos zero lactose no seu mercado local.

Em relação à análise sensorial do pudim gelado sem lactose desenvolvido neste trabalho, 50 avaliadores participaram da pesquisa e destes, $64 \%$ foram mulheres. Os participantes apresentaram idade entre 21 e 54 anos e apenas $6 \%$ dos provadores declararam ser intolerantes à lactose. Em virtude da situação de pandemia no momento da realização da análise sensorial, não foi possível selecionar os participantes da pesquisa.

O critério aparência obteve uma média de 8,04; sabor apresentou 8,35; textura, 8,20; impressão global, de 8,24; já no quesito odor, obteve-se média de 7,59, como pode ser observado na Figura 6. Através destes resultados, concluiu-se que o produto atingiu valores interessantes, demonstrando boa aceitabilidade pelos avaliadores, com todos os atributos avaliados com notas superiores a 7, que corresponde a gostei moderadamente na escala hedônica de 9 pontos.

Figura 6 - Análise sensorial com utilização de escala hedônica de 9 pontos.

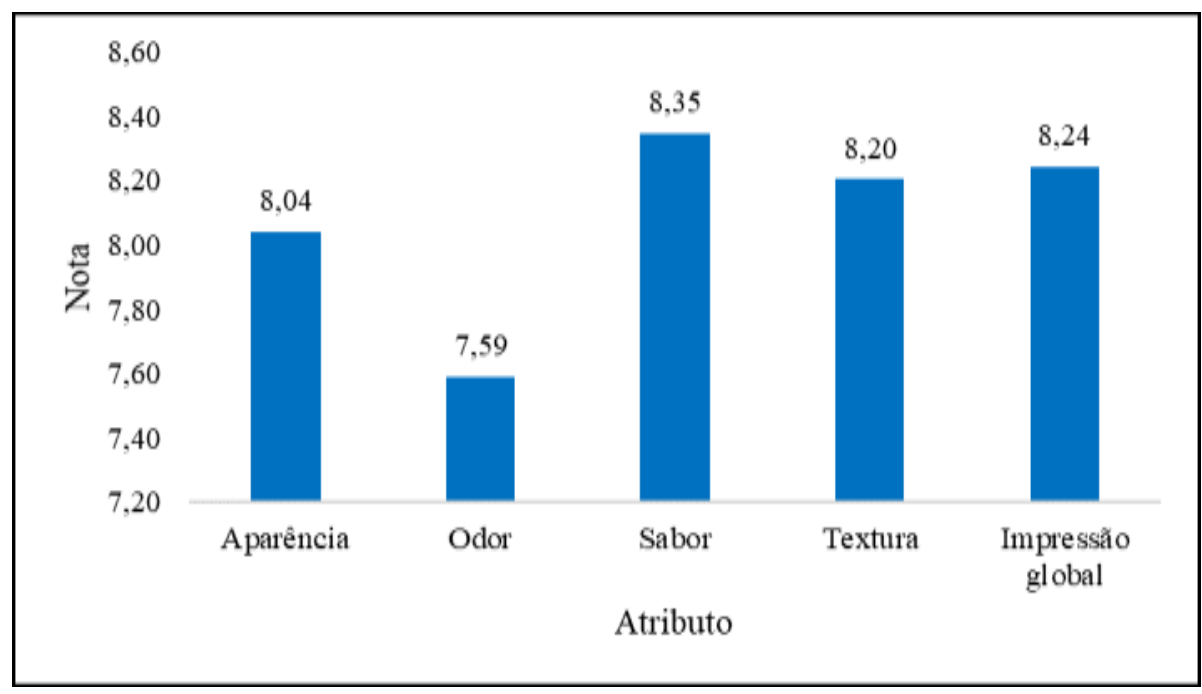

Fonte: Autores.

Silva (2019) desenvolveu formulações de gelados comestíveis tradicionais e na versão light direcionados ao público intolerante à lactose e consumidores veganos e observou que a substituição do leite tradicional pelo leite sem lactose na formulação não alterou as características do sorvete de chocolate. Seguindo a linha do desenvolvimento de sobremeses sem 
lactose, Baumgarten e Fassina (2017) avaliaram a aceitação sensorial de um bolo de caneca isento de glúten e lactose e obtiveram resultados de aceitação para os atributos de sabor e impressão global superiores quando comparados com a amostra padrão. Silva e Raupp (2017) desenvolveram um brownie funcional e a análise sensorial realizada apresentou a opção como promissora para este mercado visto que apresentou boa aceitação com relação às suas características sensoriais de textura, sabor e coloração.

Através do espaço disponível para comentários na ficha de avaliação no momento da análise sensorial, também foi possível obter a opinião dos avaliadores, que descreveram: "Adorei a consistencia e o sabor"; "Ótimo"; "Muito bom! Nem parece ser sem lactose"; "A textura Compromete" e "Não curti o limão em cima". Os comentários demonstraram que, de forma geral, o produto agradou os avaliadores, porém, alguns aspectos não satisfazeram à todos, sendo que, com maiores estudos, pode-se caracterizar como oportunidades de melhoria. Pode-se concluir que, 3 em 5 avaliadores opinaram positivamente em todos os aspectos, já 2 dos 5 avaliadores descritos opinaram medianamente para os aspectos da escala hedônica, e no espaço reservado para comentários acrescentaram que textura e aparência ainda podem ser melhorados.

Em relação a intenção de compra avaliada, observou-se que 59\% dos avaliadores responderam que certamente comprariam esse produto, como pode ser observado na Figura 7 e considerando as respostas para a opção de "Provavelmente compraria", juntas, as duas opções correspondem a $92 \%$ de avaliadores que certamente ou provavelmente comprariam o produto apresentado nesta pesquisa. Apenas $4 \%$ responderam que provavelmente não comprariam o produto. Segundo Franco (2017), a intenção de compra é um processo decisório que leva em conta vários fatores, como o preço, a conveniência e o marketing do produto, porém são as características sensoriais as determinantes nesta decisão.

Figura 7 - Percentual de pessoas que declararam a intenção de compra do pudim sem lactose desenvolvido no estudo.

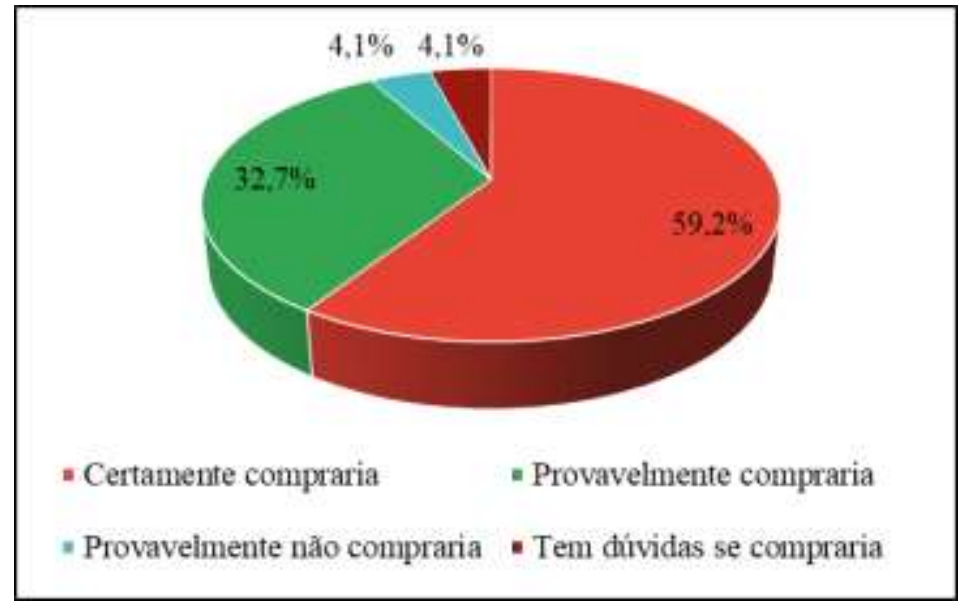

Fonte: Autores.

Tendo em vista os resultados que se mostraram muito positivos e as notas obtidas na pesquisa de intenção de compra, pode-se afirmar que $92 \%$ dos avaliadores tem intenção de adquirir o produto desenvolvido neste estudo.

Diversos estudos foram realizados buscando avaliar a intenção de compra dos consumidores para produto sem lactose (Silva; \& Raupp, 2017; Dal Castel; Endres; de Freitas; \& Rodrigues, 2017; Vieira et al., 2018; Da Silva et al., 2020; Alves; Moro; Clareto; Clerici; \& Moraes, 2020; Dutra; Pante; Reck; Bánkuti; \& Madrona, 2020), e evidenciaram resultados promissores, demonstrando que é um mercado com potencial de crescimento.

Buscando avaliar os custos relativos a produção de um pudim tradicional comparando-o com um pudim sem lactose, elaborou-se a Tabela 2, que apresenta os valores por ingrediente de forma unitária e considerando a quantidade necessária de cada um para a produção de uma receita de pudim. Para avaliação dos custos para produção do pudim tradicional e de um 
pudim sem lactose, consultou-se os valores dos ingredientes no site de um supermercado local, de acordo com as marcas dos produtos utilizados na produção dos pudins.

Tabela 2 - Valores de cada ingrediente para produção de uma receita de pudim.

\begin{tabular}{cccccc}
\hline Ingredientes & \multicolumn{3}{c}{ Valores unitários } & \multicolumn{2}{c}{ Valores para uma receita $^{\mathbf{1}}$} \\
& Quantidade & Tradicional & Sem lactose & Tradicional $^{2}$ & Sem lactose $^{\mathbf{2}}$ \\
\hline Leite condensado & $395 \mathrm{~g}$ & 5,99 & 6,29 & 11,98 & 12,58 \\
Creme de leite & $200 \mathrm{~g}$ & 2,99 & 3,29 & 5,98 & 6,58 \\
Açúcar Refinado & $1 \mathrm{~kg}$ & 3,79 & 3,79 & 0,95 & 0,95 \\
Limão Siciliano & $1 \mathrm{~kg}$ & 2,29 & 2,29 & 1,17 & 1,17 \\
Gelatina sem sabor & $20 \mathrm{~g}$ & 6,59 & 6,59 & 10,98 & 10,98 \\
Total & & - & - & 31,06 & 32,26 \\
\hline
\end{tabular}

${ }^{1}$ Valores consultados no site do Brasão Supermercados - Chapecó/SC, em 05/04/2021.

2 Valores calculados de acordo com a quantidade necessária para a elaboração de uma receita.

Fonte: Autores.

Considerando que cada receita produz, em média, 18 pudins de $30 \mathrm{~g}$ cada e a quantidade de cada ingrediente para a produção de uma receita apresentada na Tabela 1 , chegou-se ao custo de $\mathrm{R} \$ 1,73$ para um pudim tradicional e de $\mathrm{R} \$ 1,79$ para uma unidade de pudim sem lactose. Dessa forma, observa-se que há pouca diferença entre o valor de uma unidade de pudim tradicional quando comparado com a opção sem lactose, demonstrando que é possível obter-se um produto sem lactose por um valor muito próximo ao custo de um produto tradicional atualmente, proporcionando uma opção de sobremesa diferenciada que atenda ao público intolerante á lactose ou que por preferência, deseje consumir este produto.

Desta forma, ressalta-se que existe um forte desejo por parte da indústria, de desenvolver produtos sem lactose que sejam nutricionalmente adequados, econômicos e bem aceitos pelos consumidores, garantindo atributos sensoriais e nutricionais e em conformidade com as diretrizes regulatórias e econômicas em questão (Suri et al., 2019). Além disso, ressalta-se que o mercado de sobremesas, principalmente congeladas, sem lactose emerge como uma macrotendência, crescendo em virtude do apelo deste público (Silva et al., 2020a).

\section{Considerações Finais}

Através da avaliação online que esteve disponível por 14 dias, foram obtidas 521 respostas, destas 22,8\% declararam ser intolerantes a lactose. Quando perguntado sobre a dificuldade em encontrar alimentos zero lactose $53,2 \%$ responderam não encontrar facilmente e 48,6\% disseram que comprariam alimentos zero lactose, mesmo $85,2 \%$ terem respondido que este tipo de alimento possui um valor elevado no mercado da região pesquisada.

Os atributos analisados de aparência, textura, sabor e impressão global obtiveram avaliação de gostei muito, já o odor foi classificado como gostei moderadamente. A pesquisa realizada demonstrou que o pudim gelado apresentou boa aceitação pelos consumidores intolerantes a lactose e também não intolerantes, sendo que 59\% dos participantes da análise sensorial opinaram que certamente comprariam este produto. Muito embora ainda seja pouco explorado pela indústria de alimentos, os participantes se mostram interessados em seu consumo, com $92 \%$ dos avaliadores respondendo que certamente ou provavelmente comprariam o produto.

$\mathrm{O}$ produto estudado tem grande potencial de venda no mercado devido à alta aceitabilidade na análise sensorial, considerando que o custo do produto é de aproximadamente $\mathrm{R} \$ 0,06$ a mais do que para uma unidade de pudim tradicional. As 
sobremesas zero lactose ainda são pouco encontradas no comércio, e com limitada opções de compra, o que sugere que dentro de algum tempo com investimentos em pesquisa e foco neste nicho de mercado pode ser realizado um projeto piloto para avaliar a rentabilidade e aceitação desta categoria de alimento em maior escala.

\section{Referências}

Alves, I. de A., Moro, T. de M. A., Clareto, S. S., Clerici, M. T. P. S., \& Moraes, A. L. L. (2020). Sensorial analysis of gluten and lactose free muffins with reduced phenylalanin content. Research, Society and Development, 9 (12), 1-12. http://dx.doi.org/10.33448/rsd-v9i12.11126.

Baumgarten, A. C., \& Fassina, P. (2018). Análise sensorial de um bolo de caneca funcional isento de glúten e lactose. Revista Caderno Pedagógico, 14(2), 8090. http://dx.doi.org/10.22410/issn.1983-0882.v14i2a2017.1479.

Branco, M. D. S. C., Dias, N. R., Fernandes, L. G. R., Berro, E. C., \& Simioni, P. U. (2018). Classificação da intolerância à lactose: uma visão geral sobre causas e tratamentos. Revista de Ciências Médicas, 26(3), 117-125. https://doi.org/10.24220/2318-0897v26n3a3812.

BRASÃO SUPERMERCADOS. (2021). Disponível em: https://www.sitemercado.com.br/brasaochapeco/chapeco-loja-jardim-america-centro-r-sao-pedro. Acesso em: 05 br. 2021.

Dal Castel, A. P., Endres, C. M., de Freitas, A. B., \& Rodrigues, V. M. (2017). Desenvolvimento de requeijão cremoso sem lactose. Revista do Instituto de Laticínios Cândido Tostes, 72(2), 58-66. https://doi.org/10.14295/2238-6416.v72i2.560.

Dantas, A., Verruck, S., \& Prudencio, E. S. (2019). Ciência e Tecnologia de Leite e Produtos Lácteos Sem Lactose. Ponta Grossa (PR): Atena Editora. 74 p. https://doi.org/ 10.22533/at.ed.121191102.

Dekker, P. J., Koenders, D., \& Bruins, M. J. (2019). Lactose-free dairy products: market developments, production, nutrition and health benefits. Nutrients, 11(3), 551. https://doi.org/10.3390/nu11030551.

Dutra, T. V., Pante, G. C., Reck, I. M., Bánkuti, F. I., \& Madrona, G. S. (2020). Elaboração e avaliação sensorial de brownie de alfarroba isento de glúten e lactose. Research, Society and Development, 9(10), e4829108693-e4829108693. https://doi.org/10.33448/rsd-v9i10.8693.

Eiki, G., Hanai, L. N., Pires, L., Ekuni, M. M., \& Madrona, G. S. (2015). Aceitação sensorial de sorvete a base de vegetais. Revista GEINTEC-Gestão, Inovação e Tecnologias, 5(4), 2569-2578. https://doi.org/ 10.7198/S2237-0722201500040007.

Faleiros, F., Käppler, C., Pontes, F. A. R., Silva, S. S. D. C., Goes, F. D. S. N. D., \& Cucick, C. D. (2016). Uso de questionário online e divulgação virtual como estratégia de coleta de dados em estudos científicos. Texto \& Contexto-Enfermagem, 25(4). https://doi.org/10.1590/0104-07072016003880014.

Franco, L. H. E. (2017). Desenvolvimento de brownie funcional sem glúten e sem lactose. Trabalho de Conclusão de Curso, Universidade Federal do Rio Grande do Sul, Porto Alegre, RS, Brasil.

Gil, A. C. (2010). Como elaborar projetos de pesquisa. (5a ed.), Atlas. 184p.

Gille, D., Walther, B., Badertscher, R., Bosshart, A., Brügger, C., Brühlhart, M., \& Egger, L. (2018). Detection of lactose in products with low lactose content. International Dairy Journal, 83, 17-19. https://doi.org/10.1016/j.idairyj.2018.03.003.

Instituto Adolfo Lutz. (2008). Normas Analíticas do Instituto Adolfo Lutz: Métodos químicos e físicos para análise de alimentos. São Paulo: IMESP.

Lima, R. M. R., do Nascimento, K. K. B., \& de Farias, V. L. (2019). Influência do tempo de fermentação nas características físico-químicas e microbiologicas de Kefir. Brazilian Applied Science Review, 3(6), 2364-2373. https://doi.org/10.34115/basrv3n6-006.

Luthy, K. E., Larimer, S. G., \& Freeborn, D. S. (2017). Differentiating Between Lactose Intolerance, Celiac Disease, and Irritable Bowel Syndrome-Diarrhea. The Journal for Nurse Practitioners, 13(5), 348-353. https://doi.org/10.1016/j.nurpra.2017.01.018.

Matias, A. P., da Silveira, R. B., \& Brandão, M. M. (2015). Envolvimento do consumidor no processo de desenvolvimento de produtos como medida de sucesso: um estudo com empresas participantes dos prêmios FINEP e Nacional de Inovação. RAI Revista de Administração e Inovação, 12(1), 174-200.

Silva, A. C. M. L. (2019). Perfil sensorial clássico, dinâmico e direcionadores de preferências de gelados comestíveis sabor chocolate de baixa caloria nas versóes tradicional, lactose-free e vegana. Tese de doutorado em Alimentos e Nutrição, Universidade Estadual de Campinas, Campinas, SP, Brasil.

Silva, G. A., Costa, K. A., \& Giugliani, E. R. (2016). Alimentação infantil: além dos aspectos nutricionais. Jornal de Pediatria, 92(3), 2-7. https://doi.org/10.1016/j.jped.2016.02.006.

Silva, J. M., Ferreira, G. C. A., de Mesquita Oliveira, C., de Oliveira Gomes, B., Mendes, A. C., Santos, O. O., \& Pimentel, T. C. (2020a). Sobremesas de base não láctea: uma revisão da literatura. Research, Society and Development, 9(11), e77691110275-e77691110275. https://doi.org/10.33448/rsd-v9i11.10275.

Silva, J. M., Klososki, S. J., Silva, R., Raices, R. S. L., Silva, M. C., Freitas, M. Q., \& Pimentel, T. C. (2020b). Passion fruit-flavored ice cream processed with water-soluble extract of rice by-product: What is the impact of the addition of different prebiotic components? $L W T, 128$, 109472 . https://doi.org/10.1016/j.lwt.2020.109472.

Silva, L. L. D. P., \& Raupp, D. S. (2017). Desenvolvimento e aceitabilidade de brownie funcional. Revista Nutrir, 1(8), 1-22.

Suri, S., Kumar, V., Prasad, R., Tanwar, B., Goyal, A., Kaur, S., \& Singh, D. (2019). Considerations for development of lactose-free food. Journal of Nutrition \& Intermediary Metabolism, 15, 27-34. https://doi.org/10.1016/j.jnim.2018.11.003. 
Research, Society and Development, v. 10, n. 6, e49410615653, 2021

(CC BY 4.0) | ISSN 2525-3409 | DOI: http://dx.doi.org/10.33448/rsd-v10i6.15653

Uggioni, P. L., \& Fagundes, R. L. (2006). Tratamento dietético da intolerância à lactose infantil: teor de lactose em alimentos. Higiene Alimentar, 21(140), 2429.

Vieira, A. F., Rocha, A. P. T., Santos, D., Morais, H., Almeida, R. D., \& Silva, S. (2018). Aceitabilidade e caracterização física e físico-química de doce tipo doce de leite produzido com extrato hidrossolúvel de soja. Revista Principia, (42), 120-127. http://dx.doi.org/10.18265/1517-03062015v1n42p120-127.

Zenebon, O., Pascuet, N. S., \& Tiglea, P. (2008). Métodos físico-químicos para análise de alimentos. Instituto Adolfo Lutz. 1020 p. 